\title{
Avaliação do desempenho em agilidade na gestão de projetos
}

\author{
Luís Fernando Magnanini de Almeida ${ }^{\mathrm{a} *}$, Edivandro Carlos Conforto $^{\mathrm{b}}$, Sérgio Luis da Silva ${ }^{\mathrm{a}}$, \\ Daniel Capaldo Amaral ${ }^{\mathrm{b}}$ \\ aUniversidade Federal de São Carlos, São Carlos, SP, Brasil \\ bIntegrated Engineering Group - El2, São Carlos School of Engineering, Universidade de São Paulo, São Carlos, SP, Brasil \\ *luisfernando@dep.ufscar.br
}

\begin{abstract}
Resumo
Os estudos sobre gerenciamento ágil ainda não foram capazes de evidenciar a relação direta entre práticas ágeis e desempenho do projeto. A hipótese é que a combinação adequada de práticas e fatores críticos organizacionais podem levar a uma maior agilidade na gestão e, consequentemente, melhor desempenho no projeto. Este artigo apresenta uma avaliação empírica inicial dessa hipótese. Avalia-se desempenho em agilidade em duas equipes de uma mesma organização, e que, a priori, são usuárias de abordagens distintas de gestão de projetos: tradicional e ágil. Os resultados indicam que práticas como planejamento iterativo, uso de artefatos visuais para gestão e envolvimento ativo do cliente estão positivamente relacionadas com o melhor desempenho em agilidade. Há evidências de que fatores críticos de agilidade, como disponibilidade do cliente e colocalização da equipe, podem contribuir para maior agilidade e para maximizar os resultados do uso de práticas do gerenciamento ágil.
\end{abstract}

Palavras-chave

Gerenciamento ágil de projetos. Gerenciamento de projetos inovadores. Avaliação da agilidade.

\section{Introdução}

Algumas mudanças no cenário produtivo contemporâneo, como menores lead time de produção e de desenvolvimento de produtos, maior busca e importância da inovação, redução do ciclo de vida dos produtos e mudanças constantes de requisitos dos clientes (Chiesa \& Frattini, 2007), levam várias empresas a competir por meio de projetos complexos e inovadores, adotando processos de desenvolvimento flexíveis, de modo a atuarem em ambientes de negócio desafiadores e imprevisíveis.

Esses novos ambientes e as formas de enfrentá-los tiveram impacto no gerenciamento de projetos (GP). Vários autores argumentam que as ferramentas e técnicas tradicionais de GP não são adequadas a esse novo contexto (Dawson \& Dawson, 1998; Suikki et al., 2006). As principais dificuldades estão nas atividades relacionadas ao planejamento e controle de projetos (Andersen, 1996; Dvir \& Lechler, 2004;
Rozenes et al., 2004), principalmente nos que envolvem inovação e instabilidades do mercado, ou seja, nos projetos desenvolvidos nos ambientes conhecidos como "dinâmicos" ou "turbulentos".

Visando uma melhor gestão de projetos nesses ambientes, diversas propostas surgiram, autodenominadas "métodos ágeis". As mais disseminadas e conhecidas são: Scrum (Schwaber, 2004), Lean Software Development (Poppendieck \& Poppendieck, 2003), Crystal (Cockburn, 2004), Feature Driven Development - FDD (Palmer \& Felsing, 2002), Adaptive Software Development (Highsmith, 2000), Dynamic System Development Method - DSDM (Stapleton, 1997), Extreme Programming (Beck, 1999), Iterative and Visual Project Management Method - IVPM2 (Conforto \& Amaral, 2010).

Amaral et al. (2011, p. 21) definem o gerenciamento ágil de projetos (GAP) como 
[...] uma abordagem fundamentada em um conjunto de princípios, cujo objetivo é tornar o processo de gerenciamento de projetos mais simples, flexível e iterativo, de forma a obter melhores resultados em desempenho (tempo, custo e qualidade), menos esforço em gerenciamento e maiores níveis de inovação e agregação de valor para o cliente.

Ainda, segundo esses autores, as principais diferenças entre a abordagem de GP tradicional e 0 GAP seriam a autogestão, o envolvimento do cliente e simplicidade, o uso da visão no lugar do escopo e a iteração.

Apesar de a teoria sobre o GAP ter evoluído nos últimos anos, bem como sua efetiva aplicação, os ganhos em agilidade provenientes não foram demonstrados empiricamente de forma satisfatória. Isso ocorre devido à subjetividade do conceito agilidade e as dificuldades daí decorrentes, o que desafia a construção de uma medida robusta e confiável desse desempenho. A maioria dos autores que desenvolveram os métodos ágeis contemporâneos parte do pressuposto de que a agilidade é inerente à utilização das práticas propostas no gerenciamento ágil de projetos.

Algumas tentativas de se medir a agilidade foram realizadas na gestão de projetos de desenvolvimento de software (Qumer \& Henderson-Sellers, 2008; Mafakheri et al., 2008), contudo elas ainda apresentaram problemas como: foco exclusivo no desenvolvimento de software, desconsiderar fatores do ambiente de projetos e apresentar demasiada subjetividade nos seus construtos e variáveis. Logo, não foram encontrados na literatura modelos robustos para a medição da agilidade.

Com o objetivo de reduzir essa lacuna, os autores desenvolveram um modelo de agilidade para o gerenciamento de projetos cujo diferencial é contemplar tanto o desenvolvimento de produtos físicos quanto de software. 0 modelo foi construído a partir de revisão bibliográfica sistemática (RBS) que considerou diversas áreas - software, desenvolvimento de produtos, manufatura, cadeia de suprimentos e organizações -, as quais compartilham o conceito de agilidade, combinada com estudos de caso múltiplos em uma empresa de tecnologia da informação, que possui departamentos que utilizam as abordagens de gerenciamento de projetos tradicional e ágil.

Esse modelo de agilidade considera aspectos da empresa e do ambiente de negócios, denominados de fatores críticos de agilidade (FCA), os quais impactam tanto na utilização das práticas quanto o desempenho de agilidade, utilizando o resultado do levantamento de Almeida et al. (2012).
0 modelo procura, portanto, solucionar uma parte das dificuldades encontradas na teoria. 0 presente artigo tem por objetivo realizar uma primeira avaliação desse modelo proposto, por meio de um levantamento estatístico em duas equipes de projetos, pertencentes a um mesmo grupo de pesquisa, mas que são usuárias de abordagens gerenciais distintas. Essa população é de interesse para a avaliação desse modelo porque 0 ambiente e tipo de projeto são semelhantes, portanto, a principal distinção está na abordagem de gestão utilizada. Desse modo, o efeito seria mais facilmente percebido, mesmo com pequeno número de dados, permitindo uma verificação inicial do modelo teórico proposto.

Os resultados desse estudo contribuem para a exploração de novas ideias e traz novos questionamentos, além de serem fundamentais para o desenvolvimento de uma ferramenta eficaz para um estudo mais amplo com foco em ambientes empresariais e com diversos tipos de projetos.

\section{Dificuldades em se medir a agilidade no gerenciamento de projetos}

A dificuldade em medir a agilidade não é exclusiva do gerenciamento de projetos, estando presente em diversas outras áreas que também se preocupam com esse desempenho como, por exemplo, manufatura, organizações e cadeia de suprimentos (Sharifi \& Zhang, 1999; Vázquez-Bustelo et al., 2007; Driva et al., 2000; Lin et al., 2006; Yusuf et al., 2004). Essa dificuldade é decorrente do fato de que parte das definições do termo agilidade são muito genéricas e subjetivas, dificultando a sua operacionalização.

Observando a área de GP, que é o foco desse estudo, se destacam duas tentativas de medição da agilidade em projetos de desenvolvimento de software. A primeira delas foi realizada por Qumer \& Henderson-Sellers (2008), que denominaram seu modelo de 4-DAT. Esse modelo foi aplicado na comparação de 6 métodos de GAP e 2 gerenciados da forma tradicional. Para isso, ele foca em quatro perspectivas: (1) escopo do método de gestão utilizado; (2) caracterização da agilidade; (3) caracterização dos princípios do GAP e (4) caracterização do processo do software. 0 4-DAT possui quatro dimensões avaliadas: velocidade, flexibilidade, aprendizado e resiliência, e adequação aos princípios do lean.

Dentro da primeira perspectiva, o escopo do método, os autores analisam aspectos como o tamanho do time do projeto, estilo de desenvolvimento e do código (software), cultura, ambiente tecnológico e mecanismos de abstração, todos de forma qualitativa. A segunda, caracterização da agilidade, é a única que observa aspectos quantitativos, focando no processo 
e práticas utilizadas. Para isso, usa um algoritmo que analisa o número de fases, ferramentas e atributos. A terceira perspectiva avalia "princípios ágeis", de acordo com manifesto para o desenvolvimento ágil de software. A última dimensão procura avaliar o processo de desenvolvimento de software segundo as quatro dimensões do modelo.

Pela própria estrutura do 4-DAT, fica evidente que ele é restrito ao desenvolvimento de software, não podendo ser utilizado para projetos de produtos de forma ampla. Outra restrição do modelo está no uso de questões amplas como, por exemplo, "o método dá suporte a times grandes e pequenos?" Também possui muitas variáveis subjetivas, como as que avaliam se o código é "claro" e "simples".

A única perspectiva quantitativa do modelo também apresenta limitações, pois parte do pressuposto de que as práticas do GAP levam à agilidade. Ela também não observa a adequação ao tipo de projeto e utiliza uma escala binária (exemplo: tem ou não tem a prática), desse modo, não avalia a forma como a prática gerencial é realizada.

Outra tentativa de medição da agilidade em desenvolvimento de software é apresentada por Mafakheri et al. (2008). 0 modelo proposto pelos autores tenta lidar com o problema da subjetividade dos conceitos analisados por meio do uso de lógica fuzzy. 0 modelo apresenta 6 dimensões: (1) dinamismo, (2) tamanho do time, (3) comunicações, (4) teste, (5) conhecimento e habilidades desenvolvidas e (6) cultura.

Os autores aplicam o método apenas a um estudo de caso, o que não permite sua generalização. Também não consideram fatores ambientais que podem impactar na relação das práticas com o desempenho; realizam muitas estimativas para os cálculos que, adicionadas à subjetividade dos conceitos envolvidos e das dimensões escolhidas para o modelo, podem induzir ao erro nas análises. Por fim, os autores também não explicam como chegaram à fórmula geral utilizada na análise e nem o porquê de cada variável presente e dos critérios para atribuição de pesos.

Essas duas propostas de modelos de medição de agilidade no gerenciamento de projetos evidenciam suas principais dificuldades: a subjetividade no conceito e nas variáveis escolhidas, a necessidade de se considerarem aspectos ambientais e a dificuldade de se coletarem dados necessários para uso de tais modelos.

Inspirado em resolver esses problemas, a equipe propôs um modelo de agilidade para o gerenciamento de projetos, a partir do desenvolvimento de vários trabalhos específicos de pesquisa. 0 modelo inova ao conceber um parâmetro de desempenho da equipe, o seu nível de agilidade. Esse conceito estaria diretamente relacionado com as práticas e seria um dos componentes-chave para explicar o desempenho do projeto como um todo. Se este modelo estiver correto, é possível dar uma nova perspectiva aos estudos da área, melhorando a precisão das medidas que relacionam prática com desempenho no caso da abordagem de gerenciamento ágil.

\section{Modelo de agilidade para o gerenciamento de projetos}

0 modelo avaliado é fruto de um conjunto de pesquisas realizadas pela equipe cujos detalhes estão em Almeida (2012). Nesse processo, foram desenvolvidas três versões do modelo de agilidade de forma evolutiva e iterativa. Este artigo trata da avaliação da última versão do modelo em duas equipes de um grupo de pesquisa. Esta é uma etapa crucial para a melhoria do modelo e base para a uma avaliação futura no ambiente empresarial. A Figura 1 mostra as etapas para construção e melhoria do modelo que culmina nesse teste empírico.

1 - Revisão bibliográfica inicial + Versão 1 do modelo de agilidade: uma primeira revisão bibliográfica foi realizada e, a partir dela e de casos de pesquisas passadas, foi concebida a primeira versão do modelo de agilidade para gerenciamento de projetos.

2 - Aplicação do modelo em um estudo de caso exploratório: esta versão 1 foi testada em uma empresa de tecnologia de informação e comunicação (TIC) que possuía duas áreas funcionais, uma que utilizava o GP tradicional e outra que utilizava o GAP.

3 - RBS práticas, fatores críticos, indicadores e definições e modelos de agilidade: as etapas 1 e 2 permitiram um melhor entendimento da teoria e da prática e, com o resultado desse aprendizado, foram verificadas lacunas no modelo. Para seu refinamento foram realizadas três revisões bibliográficas sistemáticas, utilizando a técnica do RBS Roadmap (Conforto et al., 2011), referente a cada uma dessas lacunas teóricas. Essas buscas tiveram focos diferentes, sendo eles: identificar as práticas de gerenciamento; fatores críticos; indicadores de agilidade, além de definições e modelos de agilidade.

4 - Versão 2 do modelo de agilidade: a partir dos resultados das revisões sistemáticas e dos resultados do estudo de caso exploratório o modelo de agilidade foi aprimorado e criou-se uma segunda versão.

5 - Aplicação do modelo em um grupo de 19 empresas inovadoras: as dimensões do novo modelo foram avaliadas, por meio de um surveyem 19 empresas que participavam de um grupo voltado para a discussão e troca de experiências sobre práticas, técnicas e 


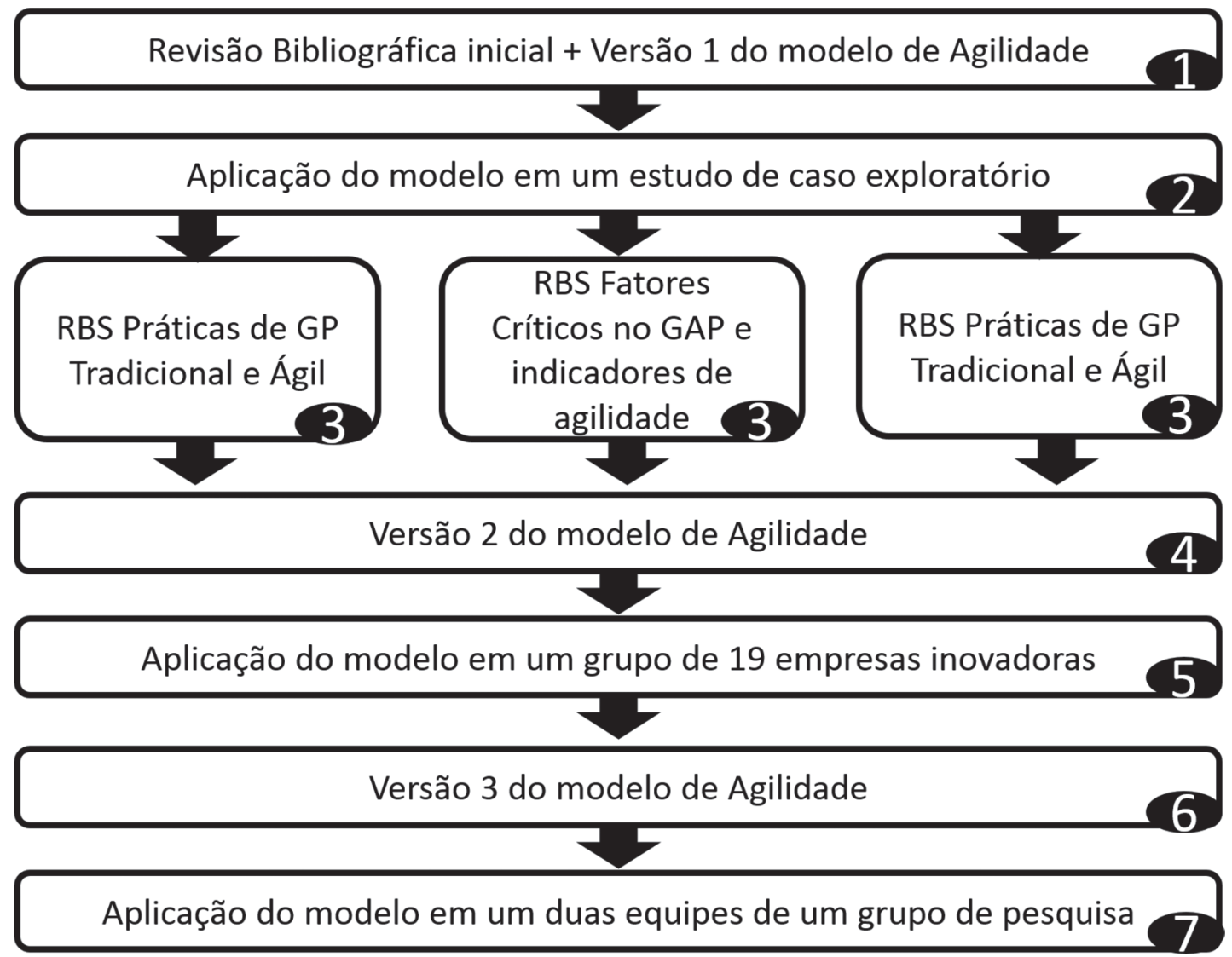

Figura 1. Etapas de pesquisa. Fonte: Elaborado pelos autores (2012).

ferramentas de inovação em desenvolvimento de produtos e projetos (Conforto et al., 2014).

6 - Versão 3 do modelo de agilidade: os resultados da survey nas 19 empresas resultaram em uma melhoria do modelo de agilidade e do questionário de pesquisa, culminando na versão 3 do modelo de agilidade. Essa conta com três dimensões que se relacionam entre si (CAs, FCAs e IAs), conforme explicado nas seções $(3.1,3.2,3.3)$ deste artigo.

7 - Aplicação do modelo em dois grupos de pesquisa: para a avaliação da versão 3 do modelo de agilidade foi realizada uma análise comparativa entre duas equipes de um grupo de pesquisa - uma que segue uma abordagem tradicional e outra a ágil, na gestão dos seus projetos de pesquisa -, sendo integrantes de um grupo com as mesmas condições ambientais (mesma universidade, local, infraestrutura, acesso a informação). Desse modo, procurou-se reduzir o impacto de causas externas que pudessem introduzir vieses na comparação entre essas duas equipes.

0 modelo (Figura 2) parte do princípio de que uma das estratégias das empresas que competem em ambientes dinâmicos de negócio é a busca da agilidade, o que está alinhado com as origens da teoria do GAP. Desse modo, a agilidade seria um desempenho necessário, mas não suficiente, para o sucesso do projeto nesses ambientes. Isso acontece porque outros fatores influenciam no desempenho final do projeto, sendo eles amplamente tratados pela literatura e denominados fatores críticos de sucesso.

A inovação desse modelo é explicitar o desempenho em agilidade e considerar que ele não é inerente às práticas propostas pelo GAP, mas sofre influência da forma como elas são utilizadas, e dos fatores críticos de agilidade presentes na organização, observando o contexto do projeto. Em função de ser uma primeira análise, esse estudo considerou as três primeiras dimensões do modelo de agilidade no gerenciamento de projetos, detalhadas nas seções 3.1, 3.2 e 3.3, com as devidas adaptações para a operacionalização, conforme justificativas apresentadas na seção 3.4 .

\subsection{Práticas/características de agilidade}

A hipótese básica do modelo de agilidade é que a utilização de certas práticas de gerenciamento levaria à agilidade do processo de GP, sendo estas 


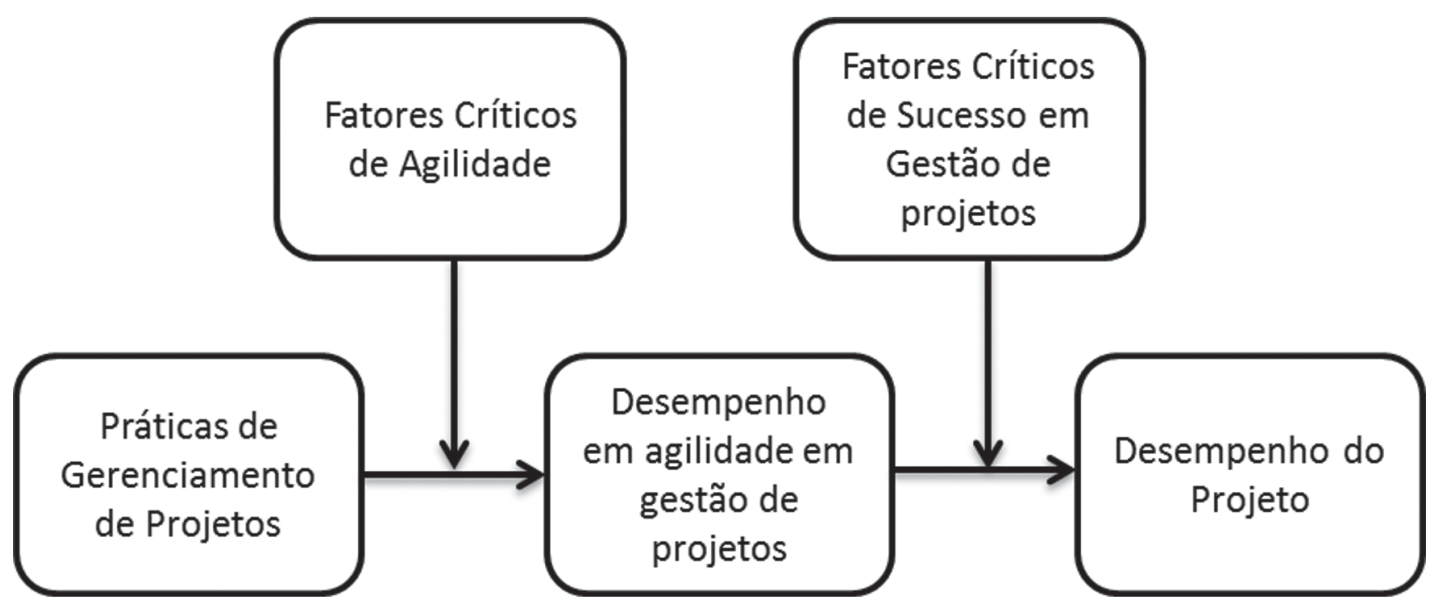

Figura 2. Modelo de agilidade para o gerenciamento de projetos. Fonte: Almeida (2012).

influenciadas por alguns fatores críticos de agilidade. As práticas são definidas como "[...] um tipo específico de ação profissional ou de gerenciamento que contribui para a execução de um processo e que pode empregar uma ou mais técnicas e ferramentas [...]" (Eder et al., 2012, p. 152).

Observando a área de gerenciamento de projetos, Eder et al. (2014) apresentam um inventário cujo objetivo é reunir e organizar as principais práticas de planejamento e controle de escopo e tempo. Segundo esses autores, inventários seriam: “[...] conjuntos união, contendo práticas, técnicas e ferramentas de ambas as abordagens, tradicional e ágil, provenientes de um estudo sistemático de livros e artigos." (Eder et al., 2012, p. 152).

Esse inventário foi construído a partir de uma revisão sistemática em bases de artigos e nos livros dos principais autores de ambas as áreas, reunindo um total de 102 práticas de GP, sendo 38 provenientes da teoria tradicional e 64 do GAP. Depois de uma análise comparativa das práticas e agrupando-as por semelhanças, esses autores destacam 18 que indicam as diferenças fundamentais de utilização das práticas entre as teorias de GP.

Contudo, para os fins desse estudo, esse número ainda era excessivo para a construção de um questionário eficiente. Sendo assim, foi realizado um novo agrupamento por semelhança e excluídos os construtos de operacionalização muito subjetivos, resultando em um total de sete diferenças fundamentais denominadas características de agilidade (CAs), sendo esse termo definido da seguinte forma:

[...] características essenciais que diferenciam as práticas provenientes do gerenciamento de projeto tradicional das do GAP, ou seja, são as características que conferem maior agilidade as práticas do gerenciamento ágil de projetos. (Almeida, et al., 2012, p. 56).

Desse modo, essas diferenças foram operacionalizadas em sete características de agilidade avaliadas neste estudo: 1) artefatos da visão, 2) conteúdo da visão, 3) o uso de iteração, 4) a priorização pelo cliente no planejamento, a 5) frequência de encontros entre o gerente e a equipe de projetos, 6) o uso de artefatos visuais no controle, e 7) os indicadores de progresso voltados a resultados tangíveis. Essas são descritas em detalhe na seção 4.2.

\subsection{Fatores críticos de agilidade}

Outra parte do modelo de agilidade considerada nesse estudo são os fatores críticos de agilidade (FCAs), sendo que eles advêm de uma adaptação do conceito de fatores críticos de sucesso. Os FCAs seriam definidos como:

\section{[...] fatores internos ou externos à organização que estão relacionados direta ou indiretamente com o processo de gerenciamento de projetos no desenvolvimento de produtos, podendo impactar positiva ou negativamente no desempenho de determinada prática, técnica ou ferramenta de gerenciamento de projetos [...] (Almeida et al., 2012, p. 97).}

Foram identificados ao todo 36 FCAs divididos em quatro dimensões: organização, processo, time e projeto. Como esse número seria muito grande para ser avaliado em um questionário eficiente, optou-se por fazer uma priorização tendo como critério o número de citações em estudos de desenvolvimento de produtos e gerenciamento ágil de projetos, assumindo que esses teriam maior potencial de apresentar correlação com o uso de práticas e a agilidade nesse primeiro teste 
do modelo. Os fatores utilizados na avaliação foram: 1) a experiência do líder, 2) a novidade tecnológica do produto, 3) a disponibilidade do cliente, 4) a autonomia, 5) a dedicação e 6) a disposição geográfica dos membros da equipe de projetos, conforme apresentado na seção 4.2.

\subsection{Desempenho/indicadores de agilidade}

A última dimensão do modelo avaliada nesse estudo é o desempenho em agilidade, indicado pelo conjunto de indicadores de agilidade (IAs).

Conforme discutido anteriormente, uma das dificuldades em se medir a agilidade é a subjetividade da maioria das definições. Para resolver esse problema foi realizada uma revisão sistemática da literatura e encontraram-se 63 definições de agilidade e 47 de flexibilidade (conceito mais próximo de agilidade para o desenvolvimento de produtos) e cunhada uma definição própria que é a adotada neste trabalho. Uma simplificação desta definição é apresentada como: "a habilidade da equipe para mudar o plano do projeto de forma contínua e rápida em função das necessidades dos clientes e mercado para continuamente adicionar valor em um ambiente de negócios inovador e dinâmico."

Para operacionalizar a medição do conceito de agilidade, conforme definição adotada neste trabalho, foram escolhidos os seguintes indicadores, derivados diretamente das definições: 1) frequência de entrega de resultados, 2) frequência da interação com o cliente, 3) tempo dispendido para tomada de decisão, 4) tempo dispendido para atualização de alguma alteração no projeto. Outro indicador (momento de congelamento do conceito do produto) foi observado em campo, mas foram constatadas falhas em sua operacionalização e o mesmo foi retirado da análise. 0 processo de escolha e estruturação desses indicadores é detalhado na seção 4.2.

\subsection{Adaptação do modelo de agilidade no gerenciamento de projetos para a pesquisa}

As seções 3.1, 3.2 e 3.3 analisaram as três dimensões do modelo de agilidade para o gerenciamento de projetos e introduziram conceitos como as características, fatores críticos e indicadores de agilidade. Algumas adaptações foram necessárias para tornar o modelo operacional e viável para pesquisa, sem, no entanto, impactar na sua essência. A análise de correlação foi escolhida ao invés da interveniência, como proposta originalmente no modelo, pois a última necessitaria de um grande número de respondentes para ser comprovada. Desse modo, havendo comprovação da relação entre as dimensões,

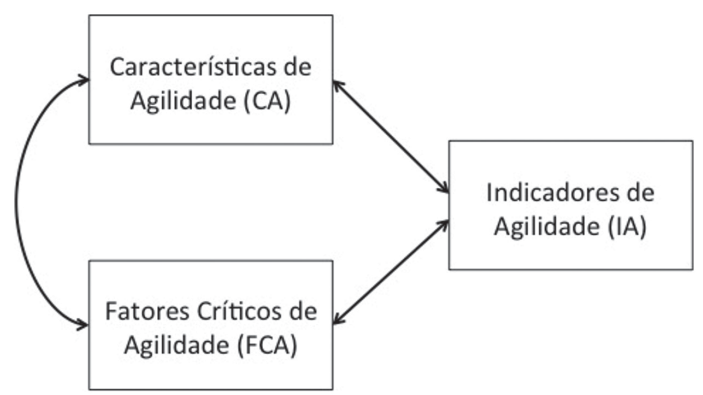

Figura 3. Modelo de agilidade adaptado para utilização na pesquisa. Fonte: Almeida (2012).

já seria um forte indício de que a interveniência poderia ocorrer e de que, essas dimensões, de fato, guardam relações entre si. Sendo assim, o modelo adotado nesta pesquisa avalia a correlação entre as características, fatores críticos e indicadores de agilidade, conforme apresentado na Figura 3.

Logo, procura-se realizar um teste preliminar da existência dessas relações, sem, no entanto, quantificar a agilidade do processo de forma absoluta, pois a criação de uma escala para esse desempenho necessitaria de mais estudos - que serão realizados no futuro -, a fim de identificar quais indicadores seriam mais importantes e para a correta atribuição de pesos a cada um. Da mesma forma, esses resultados contribuirão para um teste do modelo em um survey empresarial, a fim de comprovar a existência das relações propostas na prática.

\section{Método de pesquisa}

0 método escolhido para a pesquisa foi o levantamento estatístico (survey), pois ele permite análises empíricas, sendo realizadas as análises de correlação e comparativas entre os grupos. Desse modo, foi escolhida uma população de interesse e enviado o questionário criado para operacionalizar a pesquisa por meio eletrônico. A confecção do instrumento de pesquisa e escolha da amostra estão detalhadas nesta seção.

\subsection{População e amostra}

A população escolhida para o teste do modelo era formada por pesquisadores do Grupo de Engenharia Integrada, o qual possui membros de duas universidades públicas. 0 grupo se divide em duas equipes de pesquisa que compartilham a mesma infraestrutura. Uma dessas equipes utiliza o GAP como forma de gerenciamento de seus projetos de pesquisa e a outra usa o GP tradicional. 
A abordagem de gestão difere devido ao histórico e formação dos orientadores responsáveis por cada equipe, de modo que não foram providenciados treinamentos ou outras ações que impactassem no desempenho dos seus integrantes. Os projetos de pesquisa foram escolhidos como unidade de análise, devido a sua similaridade com os projetos de desenvolvimento de produtos inovadores (ambos complexos e com certo grau de novidade).

Foram enviados 14 questionários, dos quais $12(86 \%)$ apresentaram respostas válidas (preenchidos completamente). Dos questionários válidos metade declarou utilizar práticas provenientes da teoria tradicional de GP e a outra metade utilizava práticas de gerenciamento ágil, o que permitiu conduzir uma análise comparativa entre os dois grupos, conforme classificação apresentada na Tabela 1.

\subsection{Construção do questionário}

0 questionário foi dividido em cinco seções, agrupando as questões por assunto e ordenando-as de forma lógica para facilitar o preenchimento, sendo elas: instruções e dados para envio de relatório executivo (4 questões), caracterização do projeto escolhido para análise e do respondente (6 questões), planejamento (4 questões), controle (5 questões) e equipe do projeto (5 questões).

É importante ressaltar que as questões de cada dimensão (apresentadas no item 3 - modelo de agilidade - deste artigo) estavam distribuídas pelas diferentes seções de modo a impedir que os

Tabela 1. Amostra utilizada na pesquisa.

\begin{tabular}{lcc} 
Atividade & $\begin{array}{c}\text { Gerenciamento } \\
\text { tradicional/Equipe 1 }\end{array}$ & $\begin{array}{c}\text { Gerencimento ágil/ } \\
\text { Equipe 2 }\end{array}$ \\
\hline Mestrando & 2 & 2 \\
Doutorando & 3 & 2 \\
Doutor & 1 & 2 \\
Total & 6 & 6 \\
\hline
\end{tabular}

Fonte: Elaborado pelos autores (2012). respondentes pudessem perceber a existência de um relacionamento entre elas ou a forma de avaliação dessas dimensões. Utilizou-se escala Likert de seis pontos. As escalas foram concebidas de tal forma que um extremo (menor valor) representasse a teoria de GP tradicional e o outro extremo (maior valor) a teoria de gerenciamento ágil de projetos. No entanto, algumas questões tiveram suas escalas invertidas propositadamente para a coleta de dados. Logo, quanto mais próximo dos extremos maior era a aderência às propostas de uma das teorias.

0 questionário também possuía perguntas para a caracterização da empresa, projeto e respondente, conforme a Tabela 2. Apesar de todos respondentes participarem de um grupo de pesquisa, as questões relacionadas à empresa foram mantidas a fim de verificar se sua construção era ideal para o uso em campo, em um futuro levantamento com foco empresarial.

Essas questões, além de fornecer informações para a análise descritiva, tinham por objetivo garantir que os respondentes tivessem conhecimento suficiente para fornecer os dados necessários sobre os projetos escolhidos para análise. Perguntou-se, por exemplo, o papel do respondente no projeto, a duração do projeto, a natureza do produto resultante etc. Da mesma forma, havia questões que abordavam o segmento e o porte da empresa (no caso, a universidade), de forma a verificar se as questões estavam claras e aptas a serem utilizadas em pesquisas futuras.

A Tabela 3 traz as sete questões referentes à dimensão características de agilidade (CAs), estando elas distribuídas entre as seções de planejamento e controle.

Algumas instruções foram repassadas verbalmente aos participantes de forma a adaptar o questionário padrão ao ambiente de pesquisa, por exemplo: o pesquisador era o equivalente ao gerente (no caso de pesquisas em grupo o coordenador assumia esse papel); resultados tangiveis poderiam ser considerados algum documento formal; o produto era o resultado da pesquisa e o cliente poderia ser considerado o orientador.

Tabela 2. Construto, variáveis, código e número da questão e escala para a caracterização da empresa, projeto e respondente.

\begin{tabular}{|c|c|c|c|}
\hline Construto & Variáveis & $\begin{array}{l}\text { Código da questão } \\
\text { (número) }\end{array}$ & Escala \\
\hline Segmento de atuação & Classificação dos tipos de empresas & Firm-Sec (2) & Qualitativa ordinal nominal \\
\hline Porte da empresa & Quantidade de funcionários & Firm-size (1) & Qualitativa ordinal intervalar \\
\hline $\begin{array}{l}\text { Função/Cargo ocupado pelo } \\
\text { respondente na empresa estudada }\end{array}$ & Função desempenhada pelo respondente & Job-Role (3) & Qualitativa ordinal nominal \\
\hline Duração do projeto & Duração do projeto (em meses) & Proj-Size (6) & $\begin{array}{l}\text { Quantitativa métrica } \\
\text { (número) }\end{array}$ \\
\hline $\begin{array}{l}\text { Método de gerenciamento } \\
\text { utilizado }\end{array}$ & Declaração pela opção de um tipo de método & Meth-Adop (5) & Qualitativa ordinal nominal \\
\hline Tipo do projeto & Natureza do produto (físico ou software) & Proj-Res (7) & Qualitativa ordinal intervalar \\
\hline
\end{tabular}


Tabela 3. Construto, variáveis, código e número da questão e escala para as características de agilidade (CAs).

\begin{tabular}{|c|c|c|c|}
\hline Construto & Variáveis & $\begin{array}{l}\text { Código da questão } \\
\text { (número) }\end{array}$ & Escala \\
\hline \multirow{2}{*}{$\begin{array}{l}\text { Descrição do produto } \\
\text { do projeto de forma } \\
\text { abrangente (macro) }\end{array}$} & Conteúdo do documento que descreve o produto & CA-VisAr (9) & Qualitativa ordinal (Likert) \\
\hline & $\begin{array}{l}\text { Grau de detalhamento do documento (conjunto de } \\
\text { documentos) que descreve o produto }\end{array}$ & CA-VisCont (10) & Qualitativa ordinal (Likert) \\
\hline $\begin{array}{l}\text { Horizonte do plano do } \\
\text { projeto }\end{array}$ & $\begin{array}{l}\text { Foco no curto prazo, resultados a serem alcançados } \\
\text { em questão de dias ou semanas de forma iterativa }\end{array}$ & CA-ltPl (11) & Qualitativa ordinal (Likert) \\
\hline Priorização pelo cliente & Responsabilidade pela priorização das entregas & CA-PriPla (13) & Qualitativa ordinal (Likert) \\
\hline $\begin{array}{l}\text { Frequência do feedback da } \\
\text { equipe para com o gerente }\end{array}$ & $\begin{array}{l}\text { Tempo entre as verificações do plano do projeto } \\
\text { durante a execução (diária, semanal etc.) com a } \\
\text { participação presencial dos membros da equipe }\end{array}$ & CA-MeetFre (14) & Qualitativa ordinal (Likert) \\
\hline $\begin{array}{l}\text { Medição orientada para } \\
\text { resultados tangiveis }\end{array}$ & $\begin{array}{l}\text { Forma de acompanhamento do projeto - foco em } \\
\text { resultados tangiveis e mensuráveis (protótipos, } \\
\text { desenhos, simulações etc.) }\end{array}$ & CA-Proglnd (17) & Qualitativa ordinal (Likert) \\
\hline $\begin{array}{l}\text { Controle de fácil } \\
\text { visualização (gestão à vista) }\end{array}$ & $\begin{array}{l}\text { Uso de artefatos visuais no acompanhamento do } \\
\text { projeto }\end{array}$ & CA-ConArt (16) & Qualitativa ordinal (Likert) \\
\hline
\end{tabular}

Fonte: Elaborado pelos autores (2012).

Tabela 4. Construto, variáveis, código e número da questão e escala para os fatores críticos de agilidade.

\begin{tabular}{|c|c|c|c|}
\hline Construto & Variáveis & $\begin{array}{l}\text { Código da questão } \\
\text { (número) }\end{array}$ & Escala \\
\hline $\begin{array}{l}\text { Autonomia/Autogestão da equipe de } \\
\text { projetos para a tomada de decisão }\end{array}$ & $\begin{array}{l}\text { Grau de autonomia da equipe de projeto para tomar } \\
\text { decisões no projeto }\end{array}$ & FCA-TeamAut (19) & $\begin{array}{l}\text { Qualitativa ordinal } \\
\text { (Likert) }\end{array}$ \\
\hline $\begin{array}{l}\text { Dedicação da equipe principal do } \\
\text { projeto }\end{array}$ & $\begin{array}{l}\text { Grau de dedicação da equipe principal ao projeto } \\
\text { considerado }\end{array}$ & FCA-TeamDed (20) & $\begin{array}{l}\text { Qualitativa ordinal } \\
\text { intervalar }\end{array}$ \\
\hline $\begin{array}{l}\text { Proximidade dos membros da equipe } \\
\text { principal do projeto }\end{array}$ & $\begin{array}{l}\text { Localização geográfica dos membros da equipe de } \\
\text { projeto }\end{array}$ & FCA-TeamLoc (21) & $\begin{array}{l}\text { Qualitativa ordinal } \\
\text { intervalar }\end{array}$ \\
\hline $\begin{array}{l}\text { Grau de dificuldade para envolver } \\
\text { o cliente no planejamento e } \\
\text { desenvolvimento do projeto }\end{array}$ & $\begin{array}{l}\text { Grau de dificuldade para envolver } \\
\text { o cliente no planejamento e desenvolvimento do } \\
\text { projeto }\end{array}$ & FCA-ClieAv (12) & $\begin{array}{l}\text { Qualitativa ordinal } \\
\text { (Likert) }\end{array}$ \\
\hline $\begin{array}{l}\text { Tempo de experiência médio em } \\
\text { projetos semelhantes }\end{array}$ & $\begin{array}{l}\text { Tempo de experiência em cargos } \\
\text { de liderança em projetos de desenvolvimento de } \\
\text { produtos }\end{array}$ & FCA-LeadExp (4) & $\begin{array}{l}\text { Qualitativa ordinal } \\
\text { intervalar }\end{array}$ \\
\hline $\begin{array}{l}\text { Grau de conhecimento e domínio da } \\
\text { tecnologia principal do produto para a } \\
\text { execução do projeto }\end{array}$ & $\begin{array}{l}\text { Grau de novidade na tecnologia principal do } \\
\text { projeto, utilizada em sistemas e componentes } \\
\text { principais do produto }\end{array}$ & FCA-TechNew (8) & $\begin{array}{l}\text { Qualitativa ordinal } \\
\text { intervalar }\end{array}$ \\
\hline
\end{tabular}

Fonte: Elaborado pelos autores (2012).

Tabela 5. Construto, variáveis, código e número da questão e escala para os indicadores de agilidade.

\begin{tabular}{llll}
\hline \multicolumn{1}{c}{ Construto } & \multicolumn{1}{c}{ Variável } & $\begin{array}{c}\text { Código da questão } \\
\text { (número) }\end{array}$ & Escala \\
\hline $\begin{array}{l}\text { Busca contínua pela } \\
\text { identificação de dados e } \\
\text { informações do cliente/ } \\
\text { mercado }\end{array}$ & $\begin{array}{l}\text { Frequência de interações entre a equipe de projeto e o cliente/ } \\
\text { mercado para identificação de novas demandas e necessidades } \\
\text { dos clientes }\end{array}$ & 1A-Clielnt (22) & $\begin{array}{l}\text { Qualitativa ordinal } \\
\text { intervalar }\end{array}$ \\
$\begin{array}{l}\text { Rapidez na coleta de } \\
\text { dados e informações de } \\
\text { cliente/mercado sobre os } \\
\text { resultados do projeto }\end{array}$ & $\begin{array}{l}\text { Frequência de reuniões oficiais para entrega de resultados com } \\
\text { validação do cliente/representante do mercado }\end{array}$ & 1A-ResDeliv (15) & $\begin{array}{l}\text { Qualitativa ordinal } \\
\text { intervalar }\end{array}$ \\
$\begin{array}{l}\text { Rapidez para processar } \\
\text { a informação e tomar } \\
\text { decisão }\end{array}$ & $\begin{array}{l}\text { Tempo necessário para reunir a equipe principal do projeto para } \\
\text { discutir uma informação ou solicitação do cliente/mercado e } \\
\text { tomar uma decisão (aprovar mudança no plano do projeto) }\end{array}$ & 1A-DecTime (23) & $\begin{array}{l}\text { Qualitativa ordinal } \\
\text { intervalar }\end{array}$ \\
$\begin{array}{l}\text { Flexibilidade para adaptar } \\
\text { o plano do projeto (realizar } \\
\text { mudanças no projeto) }\end{array}$ & $\begin{array}{l}\text { Capacidade de absorver mudanças nas fases avançadas do } \\
\text { projeto }\end{array}$ & 1A-FreezConc (18)* & $\begin{array}{l}\text { Qualitativa ordinal } \\
\text { intervalar }\end{array}$ \\
$\begin{array}{l}\text { Rapidez para comunicar } \\
\text { as mudanças no plano de } \\
\text { projeto }\end{array}$ & $\begin{array}{l}\text { Tempo necessário para atualizar e comunicar as mudanças no } \\
\text { plano do projeto para todos os envolvidos no projeto }\end{array}$ & 1A-AtTime (24) & $\begin{array}{l}\text { Qualitativa ordinal } \\
\text { intervalar }\end{array}$ \\
\hline
\end{tabular}

* A questão 18, referente à flexibilidade para adaptar o plano do projeto (momento de congelamento do conceito), apresentou problemas na sua formulação, o que causou dificuldades na sua interpretação, sendo a mesma excluída das análises. Fonte: Elaborado pelos autores (2012). 
A dimensão fatores críticos de agilidade (FCAs) possuía questões distribuídas nas seções relativas à caracterização do projeto e respondente, planejamento e equipe de projetos, totalizando seis questões conforme ilustrado na Tabela 4.

Por fim, as questões referentes à dimensão indicadores de agilidade (IAs) estavam distribuídas nas seções referentes ao controle e equipe de projetos, totalizando cinco questões, conforme a Tabela 5.

\section{Resultados e discussões}

Nesta seção, são discutidos os resultados das análises estatísticas realizadas, a partir dos dados válidos, assim como realizada uma comparação entre as duas equipes de pesquisa estudadas.

\subsection{Análise da confiabilidade do instrumento de pesquisa}

Foi calculado o coeficiente alfa de Cronbach para testar a consistência interna do questionário, levando-se em consideração as perguntas referentes às características (CAs) e indicadores de agilidade (IAs). Esse tipo de teste não é aplicável aos fatores críticos de agilidade (FCAs), pois estes tratam de aspectos muito diferentes, como organização, pessoas, processos e produto, não sendo esperado um valor de alfa elevado relativo aos construtos dessa dimensão. Para a dimensão características da agilidade o valor do alfa de Cronbach foi de 0,833 e para os indicadores de agilidade 0,802 . Segundo Hair Junior et al. (2009), valores acima de 0,7 já podem ser considerados satisfatórios para essa análise. Vale ressaltar que o alfa foi calculado para o desempenho em agilidade (IA), excluindo-se a questão 18, pelo motivo já explicado na seção 4.2.

\subsection{Análise de correlação - CAs versus lAs}

A primeira análise conduzida foi a análise de correlações ( $R$ de Spearman) entre cada característica de agilidade e os indicadores de agilidade. Identificou-se que das 11 correlações significativas apenas nove possuem sentido teórico. As demais apresentaram correlação considerada estatisticamente significante, porém sem significado prático, portanto foram descartadas das análises. Conforme ilustrado na Tabela 6, das correlações válidas, quatro podem ser consideradas fortes e cinco foram consideradas moderadas (Hair Junior et al., 2009).

As duas correlações que não possuem significado prático são (CA-VisAr) versus (IA-ResDeliv), e (CA-MeetFre) versus (IA-Clielnt). A primeira foi descartada, pois a escolha dos artefatos da visão e da frequência de entrega de resultados é parâmetro do método, isto é, escolhida pelo gerente de projetos e equipe. Os artefatos visuais podem interferir no número de resultados entregue ou em sua qualidade, mas não na frequência dos mesmos. A frequência é um parâmetro estabelecido na implementação do método, na escolha da duração da iteração. Já a segunda, trata de duas frequências distintas, a de encontro da equipe com seu gestor e a da equipe com o cliente, sendo essas duas independentes entre si.

A CA que apresentou mais correlações com indicadores de agilidade foi o planejamento iterativo (CA-ltPI). Ele estava relacionado com a frequência de entrega de resultados $(0,64)$, interação com o cliente $(0,74)$ e tempo gasto para a atualização do plano do projeto e comunicação das mudanças $(0,81)$, indicando o potencial relacionamento entre os construtos, conforme indica os pressupostos da teoria de gerenciamento ágil de projetos.

A CA artefatos no controle (CA-ConArt) apresentou correlação com dois indicadores de agilidade - frequência de entrega de resultados $(0,58)$ e a interação com o

Tabela 6. Correlações entre as características de agilidade (CAs) e os indicadores de agilidade (IAs).

\begin{tabular}{ccccc}
\hline & \multicolumn{4}{c}{$\begin{array}{c}\text { Correlações: caracteristicas e indicadores de agilidade }(\mathrm{n}=12) \\
\text { Correlações significantes com valor de } p<0,05 \text { marcadas em negrito }\end{array}$} \\
\cline { 2 - 5 } & IA-ResDeliv & IA-Clielnt & IA-DecTime & IA-AtTime \\
\hline CA-VisAr & 0,60 & 0,72 & 0,21 & $-0,40$ \\
CA-VisCont & 0,54 & 0,19 & $-0,01$ & 0,81 \\
CA-ltPl & 0,64 & 0,74 & 0,20 & 0,16 \\
CA-PriPla & 0,39 & 0,59 & 0,02 & 0,68 \\
CA-MeetFre & 0,83 & 0,96 & 0,14 & 0,46 \\
CA-ConArt & 0,58 & 0,68 & 0,01 & 0,34 \\
CA-Proglnd & 0,36 & 0,45 & 0,08 & \\
\hline
\end{tabular}

Fonte: Elaborado pelos autores (2012). 
cliente $(0,68)$ - o que também está de acordo com o proposto na abordagem do GAP. Os artefatos utilizados na visão (CA-VisAr) tiveram relacionamento apenas com o indicador que avalia a interação entre a equipe e o cliente $(0,72)$. Esses artefatos também podem influenciar em outros aspectos não observados nesse estudo como, por exemplo, melhor qualidade de comunicação e maior alinhamento entre o cliente e equipe de projetos.

A frequência de encontros do gerente do projeto com a equipe (CA-MeetFre) apresentou correlações com a frequência de entrega de resultados parciais do projeto $(0,83)$ e com o tempo gasto para se atualizar o plano do projeto e comunicar as mudanças $(0,68)$. Esse número maior de encontros, além de permitir o feedback e tomada de decisão mais rápida por parte do gestor, também pode ter impacto na redução de documentação do projeto, uma vez que grande parte das informações é atualizada por meio verbal direto, em diálogos pessoais, dispensando a confecção de um documento formal e detalhado para tal. Por exemplo, uma prática comum no Scrum, um método ágil, são as reuniões diárias de até 15 minutos, entre gestor ou líder do projeto e os membros da equipe de projeto.

Por fim, a priorização dos clientes no planejamento (CA-PriPla) está diretamente relacionada com a interação do mesmo com a equipe de projeto $(0,59)$, o que é um resultado esperado, pois para que o cliente colabore na priorização do planejamento ele deve interagir com a equipe, seja presencialmente ou a distância.

As CAs conteúdo da visão (CA-VisCont) e indicadores de progresso voltados a resultados tangíveis (CA-Proglnd) não apresentaram relação significante com nenhum indicador de agilidade, o que não condiz com a teoria do GAP, podendo esta ser uma particularidade da população analisada ou, de fato, em certos casos não ocorrer como pressuposto na teoria, logo recomenda-se investigar em futuros estudos.

\subsection{Análise de correlação - CAs versus FCAs}

Para a análise do relacionamento das características de agilidade com os fatores críticos de agilidade, deve-se relembrar que a hipótese original teórica é uma relação de interveniência. Devido à população escolhida para este primeiro teste e pelo número de respondentes, não foi possível testar esse tipo de relacionamento. Optou-se por verificar apenas se 0 relacionamento entre as dimensões ocorria na prática.

Os FCAs de maior impacto também podem ser base para a seleção das variáveis em um futuro estudo que considere a interveniência, conforme proposto no modelo original. A Tabela 7 ilustra os resultados da análise de correlação.

Das seis correlações identificadas, cinco indicam correlação de forma direta (quatro CAs - sendo que uma se correlaciona com dois fatores): a priorização pelo cliente no planejamento (CA-PriPla), a frequência do encontro entre gerente e equipe de projetos (CA-MeetFre), o uso de artefatos visuais no controle (CA-VisAr) e os indicadores de progresso voltados a resultados tangíveis (CA-ConArt).

A única correlação negativa se deu entre a experiência do líder e o planejamento iterativo $(-0,63)$. Isso pode ser uma evidência de que a experiência não é um fator crítico para o uso de algumas práticas da teoria de gerenciamento ágil de projetos, embora seja considerada importante independentemente da abordagem adotada e merece ser explorada de forma mais detalhada em futuras investigações.

Já a disposição geográfica dos membros do time (FCA-TeamLoc) apresentou correlação com duas características de agilidade: a frequência de encontros do gerente com a equipe $(0,64)$ e o uso de artefatos visuais no controle $(0,60)$. Sendo assim, percebe-se que equipes colocalizadas facilitam a ocorrência de encontros e o uso de artefatos visuais, o que está de acordo com o preconizado na teoria de gerenciamento ágil. Por fim, a priorização pelo cliente está fortemente correlacionada com a disponibilidade

Tabela 7. Correlações entre as características de agilidade (CAs) e os fatores críticos de agilidade (FCAs).

\begin{tabular}{|c|c|c|c|c|c|c|}
\hline \multirow{2}{*}{ Variável } & \multicolumn{6}{|c|}{$\begin{array}{l}\text { Correlações: características e fatores criticos de agilidade }(\mathrm{n}=12) \\
\text { Correlações significantes com valores } p<0,05 \text {, marcadas em negrito }\end{array}$} \\
\hline & $\begin{array}{l}\text { FCA- } \\
\text { LeadExp }\end{array}$ & $\begin{array}{l}\text { FCA- } \\
\text { TechNew }\end{array}$ & $\begin{array}{l}\text { FCA- } \\
\text { ClieAv }\end{array}$ & $\begin{array}{l}\text { FCA- } \\
\text { TeamAut }\end{array}$ & $\begin{array}{l}\text { FCA- } \\
\text { TeamDed }\end{array}$ & $\begin{array}{l}\text { FCA- } \\
\text { Teamloc }\end{array}$ \\
\hline CA-VisAr & $-0,36$ & 0,09 & 0,61 & $-0,12$ & 0,44 & 0,57 \\
\hline CA-VisCont & 0,35 & $-0,51$ & 0,27 & $-0,02$ & 0,43 & 0,16 \\
\hline CA-ltPI & $-0,63$ & 0,16 & 0,38 & 0,20 & 0,36 & 0,46 \\
\hline CA-PriPla & $-0,12$ & 0,03 & 0,88 & $-0,32$ & 0,27 & 0,06 \\
\hline CA-MeetFre & $-0,29$ & 0,04 & 0,69 & 0,00 & 0,43 & 0,64 \\
\hline CA-ConArt & $-0,12$ & $-0,03$ & 0,42 & 0,23 & 0,44 & 0,60 \\
\hline CA-Proglnd & $-0,56$ & 0,40 & 0,44 & 0,07 & 0,12 & 0,23 \\
\hline
\end{tabular}

Fonte: Elaborado pelos autores (2012). 
do mesmo $(0,88)$, comprovando a teoria atual, o que pode indicar um efeito característico da forma de planejamento adotada, no qual o cliente participa ativamente do planejamento e execução do projeto.

\subsection{Análise de correlação - FCAs versus IAs}

Da mesma forma, realizou-se a análise entre os FCAs e IAs, a fim de verificar se de fato os FCAs podem impactar no desempenho em agilidade, assim como proposto no modelo. Nota-se que as seis correlações encontradas possuem sentido teórico (Tabela 8). Das correlações válidas, uma pode ser considerada forte e cinco moderadas, contudo quatro dessas estão bem próximas do valor mínimo para serem consideradas fortes $(>0,70)$.

0 time de projeto foi o fator que mais teve impacto na agilidade, tanto devido à dedicação (horas alocadas trabalhando exclusivamente no projeto) quanto com relação à disposição geográfica dos seus membros, cada um apresentando duas correlações.

A dedicação do time do projeto (FCA-TeamDed) está relacionada com a frequência de entrega de resultados $(0,68)$ e com menores tempos para tomada de decisão $(0,71)$, o que está de acordo com os pressupostos apresentados na teoria, indicando a importância de times dedicados como fundamentais para a utilização desses métodos. A disposição geográfica dos membros (FCA-TeamLoc) também está relacionada à frequência de entrega de resultados $(0,67)$ e com a interação com os clientes $(0,59)$. Outro resultado esperado foi a relação entre a disponibilidade dos clientes (FCA-ClieAv) e a interação da equipe com os mesmos $(0,69)$.

Um resultado inesperado indicou que a experiência do líder (FCA-LeadExp) se relaciona de forma inversa com o tempo necessário para a atualização $(-0,68)$. Esse resultado pode ser um indicativo de que profissionais mais experientes primam por informações mais detalhadas e por documentação mais abrangente, o que demanda maiores tempos de confecção e atualização.

Os indicadores que apresentaram um maior número de correlações são os que estariam mais propensos à influência desses fatores, como a frequência de entrega de resultados e da interação com o cliente. 0 tempo de decisão também sofreu influência do FCA dedicação do time. Por fim, o tempo de atualização não apresentou relacionamento com os fatores críticos de agilidade analisados. Isso porque ele é mais dependente da forma de gerenciamento adotada, que está relacionada aos CAs planejamento iterativo e encontro entre o gerente e a equipe de projetos, conforme apresentado na seção 5.2.

\subsection{Análise comparativa}

Para a análise comparativa adotou-se o valor da mediana entre as equipes, pois é uma medida menos tendenciosa para valores extremos do que a média. A primeira análise foi feita observando-se as características de agilidade (CAs) e a comparação entre os grupos, conforme ilustrado na Figura 4.

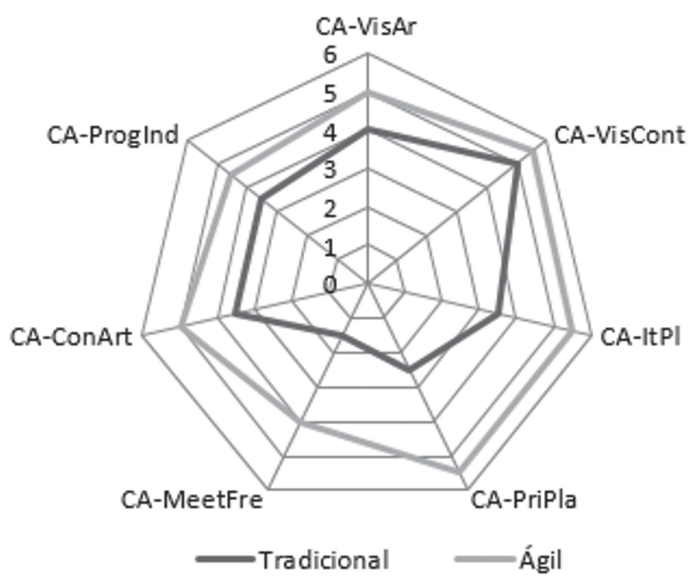

Figura 4. Comparação entre as equipes que utilizam GP tradicional e GAP com relação às características de agilidade. Fonte: Elaborado pelos autores (2012).

Tabela 8. Correlações entre os fatores críticos de agilidade (FCAs) e os indicadores de agilidade (IAs).

\begin{tabular}{ccccc}
\hline \multirow{2}{*}{ Variável } & \multicolumn{4}{c}{$\begin{array}{c}\text { Correlações: fatores criticos e indicadores de agilidade }(\mathrm{n}=12) \\
\text { Correlações significantes com valores } p<0,05, \text { marcadas em negrito }\end{array}$} \\
\cline { 2 - 5 } & IA-ResDeliv & IA-Clielnt & IA-DecTime & IA-AtTime \\
\hline FCA-LeadExp & $-0,06$ & $-0,27$ & $-0,35$ & $-0,68$ \\
FCA-TechNew & $-0,15$ & $-0,06$ & $-0,08$ & 0,20 \\
FCA-ClieAv & 0,38 & 0,69 & 0,05 & 0,15 \\
FCA-TeamAut & 0,14 & 0,01 & $-0,25$ & 0,09 \\
FCA-TeamDed & 0,68 & 0,46 & 0,71 & 0,35 \\
FCA-TeamLoc & 0,67 & 0,59 & $-0,33$ & 0,34 \\
\hline
\end{tabular}


Fica evidente a maior presença das CAs na equipe que utiliza a abordagem do gerenciamento ágil, evidenciando que esse construto teórico realmente consegue separar os grupos que utilizam práticas de $\mathrm{GAP}$ versus usuários de práticas tradicionais de GP.

Contudo, percebe-se que o grupo que utiliza o GP tradicional teve desempenho acima de três (que seria o valor que separaria as teorias) em várias CAs, o que pode indicar que mesmo práticas tradicionais, dependendo de como são empregadas, se aproximam da forma de utilização e resultados esperados pelo GAP.

Em relação aos fatores críticos de agilidade (FCAs), conforme ilustrado na Figura 5, observa-se que há diferenças significativas, mesmo se tratando de projetos de pesquisa e de equipes que utilizam mesma infraestrutura: condições ambientais semelhantes. Elas

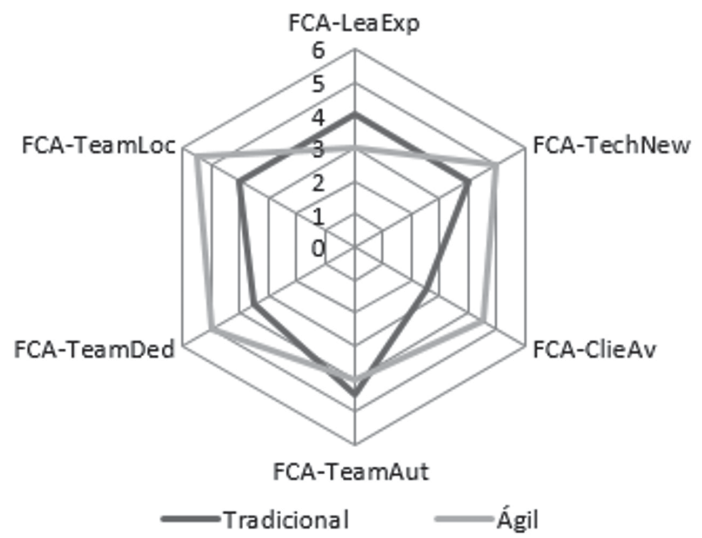

Figura 5. Comparação entre as equipes que utilizam GP tradicional e GAP com relação aos fatores críticos de agilidade. Fonte: Elaborado pelos autores (2012).

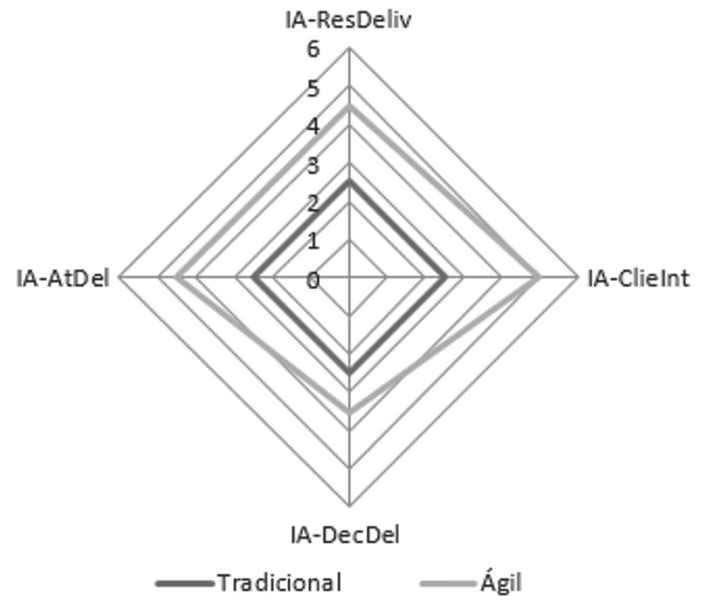

Figura 6. Comparação entre as equipes que utilizam GP tradicional e GAP com relação aos indicadores de agilidade. Fonte: Elaborado pelos autores (2012). ocorrem principalmente com relação à localização (FCA-TeamLoc), dedicação do time de projetos (FCA-TeamDed) e da disponibilidade do cliente (FCA-ClieAv), que neste caso foram considerados os orientadores acadêmicos das equipes de pesquisa, sendo este um indício de que as abordagens de gestão fazem diferença.

Existe a impressão de que os integrantes da equipe tradicional seriam mais autônomos (FCA-TeamAut) devido à menor priorização/validação do resultado com o cliente (orientador). Essa "maior" liberdade também teve impacto com relação à localização (alguns trabalhavam parte do tempo em casa). Contudo, nos momentos de avaliação desses projetos, eram solicitadas grandes modificações, o que ocasionava um maior retrabalho. Com relação à experiência do líder, o orientador responsável pela equipe de GP tradicional possuía maior tempo de atividade profissional quando comparado ao da GAP.

Por fim, quando observados os indicadores de agilidade, a equipe que adotou o GAP obteve melhor desempenho em todos os aspectos considerados, conforme ilustrado na Figura 6. A principal diferença ocorreu na interação com o cliente (orientadores).

Os resultados indicam que mesmo as equipes submetidas a influências ambientais semelhantes e realizando projetos com complexidade e grau de inovação análogos, a equipe que adotou práticas do GAP obteve melhor desempenho em todos os aspectos da agilidade considerados, em particular na interação com o cliente, tempo para tomada de decisão e atualização e entrega de resultados.

\section{Conclusões}

Os dados coletados permitiram avaliar o gerenciamento ágil de projetos, a partir de um caso especial de organização em que projetos convivem em ambientes com equipes e características similares, mas gerenciados de maneira diferente, tradicional e ágil. Isso permitiu uma comparação mais controlada dos resultados, se confrontado com as avaliações de desempenho dos métodos ágeis identificadas na teoria. Empregou-se também um modelo teórico que inova ao propor o conceito de desempenho em agilidade na gestão de projetos.

0 estudo inicia reforçando a validade das hipóteses de que o planejamento iterativo, o encontro frequente da equipe com o líder de projeto, a utilização artefatos visuais no controle do projeto e o conteúdo na descrição do produto são as características que mais se relacionam com o desempenho em agilidade. Isso reforça a teoria dado que as práticas do gerenciamento 
ágil mais conhecidas são justamente o planejamento iterativo e a visão do produto.

A segunda contribuição é evidenciar a importância de certas condições ambientais, os fatores críticos, para que as práticas de gerenciamento ágil resultem em maior agilidade. lsso foi indicado pelos resultados das correlações entre fatores críticos de agilidade e a presença das características de agilidade. 0 grau de dificuldade para envolver o cliente e a colocalização da equipe mostraram-se mais relacionados com as principais práticas ágeis considerando o contexto e projetos analisados. lsso é algo novo na teoria, pois esses fatores não foram tratados nas avaliações anteriores da teoria de gerenciamento ágil de projetos.

A comprovação de relacionamento entre as variáveis das dimensões propostas no modelo de desempenho em agilidade utilizadas na pesquisa indicam que este modelo pode estar correto e deveria ser avaliado em estudos com populações de empresas e maior quantidade de projetos e equipes. 0 modelo é reforçado pelo fato das medidas do conceito teórico características de agilidade (CAs) terem sido capazes de diferenciar as duas equipes de pesquisa que à priori já se sabia serem usuárias do gerenciamento tradicional e ágil de projetos. Isso indica que o modelo teórico, os construtos e o instrumento de pesquisa desenvolvidos são úteis. Essa é uma contribuição importante, pois podem ser utilizados como ponto de partida para novas pesquisas.

Apesar de a equipe usuária do GAP apresentar valores superiores com relação a todas as CAs, um resultado inesperado foi a alta pontuação para o grupo de usuários das práticas tradicionais. lsso remete a duas hipóteses. Hipótese 1, necessidade de aprimoramento dos instrumentos de coleta de dados para aumentar a diferença entre os grupos. Hipótese 2, algumas práticas ágeis poderiam ser utilizadas de maneira parcial e no contexto do ambiente tradicional. Ambas as hipóteses contribuem para a teoria de GP e indicam aspectos que precisam ser mais bem investigados.

Os fatores críticos de agilidade (FCAs) apresentaram semelhanças entre os grupos. Esse resultado era esperado dado que as equipes atuavam na mesma organização (mesmo ambiente de projetos e mesma infraestrutura), corroborando mais uma vez o modelo. Já com relação aos indicadores de agilidade (IAs), a equipe que utilizava o GAP apresentou maior desempenho em todos os aspectos analisados, indicando que, para esse caso, o GAP conferiu, de fato, maior agilidade ao processo de gestão. Sugere-se a repetição desse estudo em meio empresarial e com população maior e diversificada para que essa evidência seja confirmada.

Diante das várias evidências, conclui-se que o modelo adotado na pesquisa não pode ser refutado e continua válido. Ele é, portanto, aplicável e deveria ser foco de novas avaliações. É preciso verificar com mais cuidado as relações, em especial, utilizá-lo em pesquisas projetadas de forma a viabilizar a medição dos relacionamentos de interveniência.

Uma última contribuição desta pesquisa é que os construtos e instrumentos criados para a "operacionalização" da pesquisa (características de agilidade e fatores críticos de agilidade) conseguiram cumprir sua função. Desse modo, esse é um primeiro passo para a ampliação e continuação dos testes do modelo proposto. Como estudos futuros, propõe-se a repetição desse levantamento, em âmbito empresarial, para populações maiores e para projetos mais complexos, a fim de verificar se as mesmas correlações se repetem nesses ambientes, assim como a aplicabilidade do modelo para situações de ambientes organizacionais diferentes entre as equipes de projetos.

\section{Agradecimentos}

Os autores agradecem o apoio financeiro à pesquisa oferecido por Fapesp, CNPq e Capes, sem o qual não seria possível a realização deste trabalho. Agradecem também as contribuições e comentários oferecidos pelos revisores anônimos e editor da revista, que foram essenciais para a melhoria do artigo.

\section{Referências}

Almeida, L. F. M. (2012). Características, fatores críticos e indicadores de agilidade no gerenciamento de projetos de produtos inovadores (Dissertação de mestrado). Universidade Federal de São Carlos, São Carlos.

Almeida, L. F. M., Conforto, E. C., Silva, S. L., \& Amaral, D. C. (2012). Fatores críticos da agilidade no gerenciamento de projetos de desenvolvimento de novos produtos. Revista Produto e Produção, 13(1), 93-113.

Amaral, D. C., Conforto, E. C., Benassi, J. L. C., \& Araújo, C. (2011). Gerenciamento ágil de projetos - aplicação em produtos inovadores. São Paulo: Saraiva.

Andersen, E. (1996). Warning: activity planning is hazardous to your project's health! International Journal of Project Management, 14(2), 89-94. http://dx.doi.org/10.1016/02637863(95)00056-9.

Beck, K. (1999). Extreme programming explained. New York: Pearson Education, Inc.

Chiesa, V., \& Frattini, F. (2007). Exploring the differences in performance measurement between research and development: evidence from a multiple case study. $R \& D$ Management, 37(4), 283-301. http://dx.doi.org/10.1111/ j.1467-9310.2007.00476.x.

Cockburn, A. (2004). Crystal clear - a human-powered methodology for small teams (Agile Software Development Series). New Jersey: Addison-Wesley Professional.

Conforto, E. C., \& Amaral, D. C. (2010). Evaluating an agile method for planning and controlling innovative projects. Project Management Journal, 41(2), 73-80. http://dx.doi. org/10.1002/pmj.20089. 
Conforto, E. C., Amaral, D. C., \& Silva, S. L. (2011). Roteiro para revisão bibliográfica sistemática: aplicação no desenvolvimento de produtos e gerenciamento de projetos. In Anais do $8^{\circ}$ Congresso Brasileiro de Gestão de Desenvolvimento de Produtos. Porto Alegre, RS.

Conforto, E. C., Salum, F., Amaral, D. C., Silva, S. L., \& Almeida, L. F. M. (2014). Can agile project management be adopted by industries other than software development? Project Management Journal, 45(3), 21-34. http://dx.doi. org/10.1002/pmj.21410.

Dawson, R., \& Dawson, C. (1998). Practical proposals for managing uncertainty and risk in project planning. International Journal of Project Management, 16(5), 299-310. http:// dx.doi.org/10.1016/S0263-7863(97)00059-8.

Driva, H., Pawar, K. S., \& Menon, U. (2000). Measuring product development performance in manufacturing organizations. International Journal of Production Economics, 63(2), 147159. http://dx.doi.org/10.1016/S0925-5273(99)00007-9.

Dvir, D., \& Lechler, T. (2004). Plans are nothing, changing plans is everything: the impact of changes on project success. Research Policy, 33(1), 1-15. http://dx.doi.org/10.1016/j. respol.2003.04.001.

Eder, S., Conforto, E. C., Amaral, D. C., \& Silva, S. L. (2014). Diferenciando as abordagens tradicional e ágil de gerenciamento de projetos. Production, No Prelo.

Eder, S., Conforto, E. C., Schnetzler, J. P., Amaral, D. C., \& Silva, S. L. (2012). Estudo das práticas de gerenciamento de projetos voltadas para o desenvolvimento de produtos inovadores. Revista Produto e Produção, 13(1), 148-165.

Hair Junior, J. F., Black, W. C., Babin, B. J., Anderson, R. E., \& Tatham, R. L. (2009). Análise multivariada de dados. Porto Alegre: Bookman.

Highsmith, J. (2000). Adaptive software development: a collaborative approach to managing complex systems. New York: Dorset House Publishing.

Lin, C., Chiu, H., \& Chu, P. (2006). Agility index in the supply chain. International Journal of Production Economics, 101(2), 285-299. http://dx.doi.org/10.1016/j.jpe.2004.11.013.

Mafakheri, F., Nasiri, F., \& Mousavi, M. (2008). Project agility assessment: an integrated decision analysis approach.
Production Planning and Control, 19(6), 567-576. http:// dx.doi.org/10.1080/09537280802360884.

Palmer, S. R., \& Felsing, J. M. (2002). A pratical guide to feature-driven development (The Coad series). New Jersey: Prentice Hall.

Poppendieck, M., \& Poppendieck, T. (2003). Lean software development: an agile toolkit for software development managers. Boston: Addisson-Wesley Professional.

Qumer, A., \& Henderson-Sellers, B. (2008). An evaluation of the degree of agility in six agile methods and its applicability for method engineering. Information and Software Technology, 50(4), 280-295. http://dx.doi.org/10.1016/j. infsof.2007.02.002.

Rozenes, S., Vitner, G., \& Spraggett, S. (2004). MPCS: Multidimensional Project Control System. International Journal of Project Management, 22(3), 109-118. http:// dx.doi.org/10.1016/S0263-7863(03)00002-4.

Schwaber, K. (2004). Agile project management with SCRUM. Washington: Microsoft Press.

Sharifi, H., \& Zhang, Z. (1999). A methodology for achieving agility in manufacturing organization: an Introduction. International Journal of Production Economics, 62(1-2), 7-22. http://dx.doi.org/10.1016/S0925-5273(98)00217-5.

Stapleton, J. (1997). DSDM, dynamic systems development method: the method in practice. Cambridge: Cambridge University Press.

Suikki, R., Tromstedt, R., \& Haapasalo, H. (2006). Project management competence development framework in turbulent business environment. Technovation, 26(5), 723738. http://dx.doi.org/10.1016/j.technovation.2004.11.003.

Vázquez-Bustelo, D., Avella, L., \& Fernández, E. (2007). Agility drives, enablers and outcomes: empirical test of an integrated agile manufacturing model. International Journal of Operations \& Production Management, 27(12), 1303-1332. http://dx.doi.org/10.1108/01443570710835633.

Yusuf, Y. Y., Gunasekaran, A., Adeleye, E. O., \& Sivayoganathan, K. (2004). Agile supply chain capabilities: Determinants of competitive objectives. European Journal of Operational Research, 159(2), 379-392. http://dx.doi.org/10.1016/j. ejor.2003.08.022.

\section{Evaluating agility performance in innovative project management Abstract}

Studies on agile project management have been unable to demonstrate a direct correlation between agile practices and project performance. The assumption has been that the proper combination of practices and organization factors can result in greater agility and thus better project performance. In a preliminary empirical investigation of this hypothesis, this paper assesses the "agility performance" of two project teams in the same organization that are self-declared users of distinct project management approaches: traditional and agile. The results show that iterative planning, the use of visual artifacts to manage the project and active client involvement are positively correlated with agility performance indicators. In addition, evidence shows that some "Agility Critical Factors" such as client availability and team co-location may contribute to greater agility performance and maximize the results of agile management practices.

\section{Keywords}

Agile project management. Innovative project management. Agility assessment. 